\title{
PERFIL ANTROPOMÉTRICO EN ADULTOS DEL NOROESTE ARGENTINO: COMPARACIÓN CON UNA REFERENCIA INTERNAGONAL
}

\author{
Delia Lomaglio ${ }^{1 *}$, Rafael Carrillo2,3, María S. Mesa4, José E. Dipierri3 , Ignacio F. Bejarano2,3 , Jorge \\ Morales², Norma Dip¹, Marcos Mamani², Andrea Cazon², Ana Davalos², Graciela Cabrera², Estela \\ M. Román ${ }^{3}$ José L. Pacheco ${ }^{5}$ y María D. Marrodán ${ }^{4}$
}

\begin{abstract}
${ }^{I}$ Centro de Estudios de Antropología Biológica. Facultad de Ciencias Exactas y Naturales. Universidad Nacional de Catamarca. Catamarca. Argentina

${ }^{2}$ Unidad de Investigación Antropología Biológica (UIAB). Facultad de Humanidades y Ciencias Sociales. Universidad Nacional de Jujuy. San Salvador de Jujuy. Jujuy. Argentina

${ }^{3}$ Instituto de Biología de la Altura. Universidad Nacional de Jujuy. San Salvador de Jujuy. Jujuy. Argentina

${ }^{4}$ Departamento de Zoología y Antropología Física. Facultad de Ciencias Biológicas. Universidad Complutense de Madrid. Madrid. España ${ }^{5}$ Departamento de Enfermería. Escuela Universitaria de Enfermería, Fisioterapia y Podología. Universidad Complutense de Madrid. Madrid.España
\end{abstract}

\section{PALABRAS CLAVE adultos; Noroeste Argentino; perímetros; pliegues; antropometría}

\begin{abstract}
RESUMEN Desde una perspectiva bioantropológica las mediciones antropométricas en adultos son exhaustivamente utilizadas en la evaluación morfológica de poblaciones argentinas extintas. La mayoría de los estudios antropométricos en adultos argentinos contemporáneos se limitan a la evaluación de talla, peso e IMC. El objetivo de este trabajo fue describir otras variables antropométricas en adultos del Noroeste argentino (NOA) y compararlas con una referencia internacional. Los datos de peso, talla, perímetros (brazo, muslo, pierna, cintura) y pliegues (tríceps, subescapular) proceden de 881 individuos (526 mujeres; 355 varones) sanos de 20-60 años de edad de distintas localidades de Catamarca y Jujuy. Se calcularon estadísticos descriptivos (media y DS) por sexo y dos
\end{abstract}

grupos de edad: 20-39 y 40-60 años. Las comparaciones se establecieron con adultos de EEUU de origen mejicano (Centers for Disease Control, CDC 2003-2006) con la prueba t. Se calcularon los percentilos con el método LMS. En ambos sexos y grupos de edad los adultos del NOA presentaron valores significativamente inferiores a los de la referencia en todas las variables consideradas, excepto para pliegues tricipital y subescapular en varones de 40-60 años. No se han encontrado antecedentes antropométricos comparables semejantes para poblaciones argentinas y del NOA. El perfil antropométrico de los adultos del NOA difiere significativamente con respecto a la población de referencia con un origen étnico afín. Rev Arg Antrop Biol 17(1):07-18, 2015.

\section{KEY WORDS adults; Argentine Northwest; circumferences; skinfold thickness; anthropometry}

ABSTRACT From a bioanthropological point of view anthropometric measurements in adults are extensively used in the morphological evaluation of extinct Argentine populations. Most anthropometric studies in contemporary Argentine adults are limited to the assessment of height, weight and BMI. The aim of this study was to describe other anthropometric variables in northwestern Argentine (NWA) adults and to compare them with an international reference. Data on weight, height, circumferences (arm, thigh, leg, waist), and skinfolds (triceps, subscapular) come from 881 (526 women, 355 men) 20-60 year-old healthy individuals from different localities of Jujuy and Catamarca. Descriptive statistics (mean and SD)

De acuerdo al Informe de un Comité de Expertos de la Organización Mundial de la Salud (OMS, 1995) sobre el crecimiento y desarrollo en distintas etapas de la ontogénesis, se reconoce que la nutrición y la salud de los adultos representan el "sostén económico de la sociedad", no solo en las sociedades agrícolas tradicionales, sino también en los países industrializados. Basado en esta premisa, el Comité recomienda que la OMS promueva en adultos, la obtención de datos antropomé- were calculated by sex and two age groups: $20-39$ and 40-60 years. Comparisons were made with adults of Mexican origin from the United States (Centers for Disease Control, CDC 2003-2006) using the t-test. Percentiles were calculated using the LMS method. In both sexes and age groups NWA adults presented significantly lower values than those of the reference group in all the variables considered, except for triceps and subscapular skinfolds in males of 40-60 years old. There are no comparable anthropometric records for Argentine and NWA populations. The anthropometric profile of NWA adults differs significantly from that of the ethnically related reference population. Rev Arg Antrop Biol 17(1):07-18, 2015.

Financiamiento: Agencia Española de Cooperación Internacional (AECID), Proyectos: A/025549/09; A/030303/10. Secretaría de Ciencia y Técnica y Estudios Regionales (SECTER), Universidad Nacional de Jujuy, Proyecto PICTO-2008-00139. Secretaría de Ciencia y Tecnología, Universidad Nacional de Catamarca, Proyecto 02/G410.

*Correspondencia a: Delia Lomaglio. Centro de Estudios de Antropología Biológica. Luis Díaz (Norte) 267. 4700 Catamarca. Argentina. E-mail: delialomaglio@yahoo.com

Recibido 30 Diciembre 2013; aceptado 20 Mayo 2014

doi:10.17139/raab.2015.0017.01.03 
tricos apropiados de referencia internacional. Esta recomendación resulta procedente para variables antropométricas pero no para indicadores o índices antropométricos, como el índice de masa corporal (IMC), para los cuales son más confiables especialmente en adultos, los puntos de corte pragmáticamente establecidos a partir de estos indicadores (De Onis y Habicht, 1996).

En general se admite que las poblaciones humanas de distinto origen étnico, sobre todo las infantiles, siguen un patrón de crecimiento semejante cuando son expuestas a las mismas condiciones ambientales. Sin embargo, no se puede excluir que las diferencias observadas en el patrón de crecimiento entre poblaciones no sólo refleje la influencia del ambiente, sino también las diferencias en el potencial genético interétnico (Butte et al., 2007). Esta consideración refuerza la necesidad de reconocer las características antropométricas de las poblaciones de acuerdo a su procedencia étnica y/o geográfica.

También cabe señalar que el uso de la antropometría en adultos se ha centrado en la evaluación del riesgo de enfermedades crónicas asociadas al sobrepeso y a la distribución de la grasa corporal. Para ello se ha recurrido a la definición de índices e indicadores basados en variables antropométricas de fácil medición y monitoreo y aplicación universal tal como el peso y la talla. Menor atención tuvieron otras variables antropométricas como los pliegues o perímetros y su variación interpoblacional.

Desde una perspectiva bioantropológica las mediciones antropométricas han sido exhaustivamente utilizadas en la evaluación morfológica de poblaciones extintas del territorio argentino (Carnese y Pucciarelli, 2007). Excepto aquellos llevados a cabo con fines deportivos, los estudios antropométricos en adultos sanos contemporáneos argentinos son escasos (Bejarano et al., 1996, 2009, 2013; Agrelo et al., 1999; Del Valle et al., 2007; Peláez et al., 2010). Centrados en la evaluación de la prevalencia de estados de malnutrición por exceso (sobrepeso y obesidad) y en determinar factores de riesgo de comorbilidades asociadas al aumento del peso corporal (diabetes, hipertensión y enfermedad corona- ria) la mayoría de estos estudios se limitan a la evaluación de la talla, del peso y al cálculo del IMC (Hernández et al., 1987; Carbajal et al., 2001; Braguinsky, 2002; De Sereday et al., 2004; Feldstein et al., 2005).

El Noroeste argentino (NOA) es una de las dos subdivisiones administrativas que junto a la otra, el Noreste argentino (NEA) integran la región del Norte grande argentino. Es una entidad política que no se corresponde con la región histórico-geográfica del NOA. Fue creada el 9 de abril de 1999, con la integración de las provincias de Jujuy, Salta, Santiago del Estero, Catamarca y Tucumán. Sin embargo, no existe aún consenso entre los investigadores acerca de las provincias que integran el NOA. Algunos autores incluyen también a la provincia de La Rioja. Pese a ello es aceptado que las seis provincias (Jujuy, Salta, Catamarca, Tucumán, Santiago del Estero y La Rioja) mantienen una homogeneidad demográfica que justifica su inclusión en un conjunto espacial supraprovincial (Pontussi, 1995; Boleda, 1998) ya que además de las características económicas y demográficas, contribuyen también a la singularidad del NOA como región, los antecedentes históricos, etnohistóricos y culturales (Bazán, 1986). Biogeográficamente, el NOA se extiende desde los Andes al oeste hacia las regiones menos elevadas de las Yungas y del Chaco hacia el este (Pontussi, 1995) (Fig.1).

Originado a partir de esta configuración biogeográfica, sus poblaciones con un fuerte componente amerindio (Wang et al., 2008; Dipierri et al., 2011) se asientan en un gradiente altitudinal ( $>500 \mathrm{msnm}$ a $<3500 \mathrm{msnm}$ ) con variados entornos socioeconómicos y culturales. Comparada con el resto del país, el NOA conforma junto con otras provincias del NEA, el territorio más pobre con bajos niveles de actividad económica, elevados porcentajes de necesidades básicas insatisfechas, altos porcentajes de pobreza e indigencia, altas tasas de mortalidad maternoinfantil y en general, peores condiciones sanitarias (Bolsi et al., 2009).

Estudios antropométricos realizados en adultos de la provincia de Jujuy basados en la talla, peso y perímetros de la cintura y de la cadera muestran que estas poblaciones presentan indicadores de adiposidad elevados, 


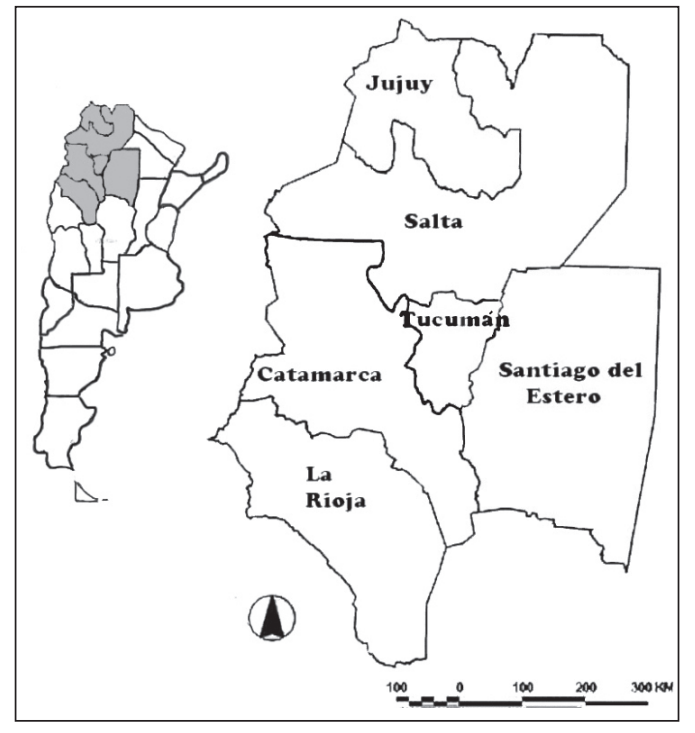

Fig. 1. Ubicación del Noroeste argentino (NOA) en la República Argentina.

propios de poblaciones con transición nutricional completa (Bejarano et al., 2013). El objetivo de este trabajo fue evaluar el estado nutricional y describir y comparar el perfil antropométrico de adultos contemporáneos de las provincias de Catamarca y Jujuy integrantes del NOA, con una referencia internacional.

\section{MATERIAL Y MÉTODOS}

Se realizó un estudio de tipo transversal en sujetos adultos, para el cual se contó con la aprobación bioética correspondiente y el consentimiento informado de los participantes. Entre julio 2010 y marzo 2011 en distintas localidades de las provincias de Jujuy ( $\mathrm{La}$ Quiaca, San Salvador de Jujuy, Palpalá) y Catamarca (San Fernando del Valle de Catamarca) se evaluaron antropométricamente 881 individuos, 526 mujeres $(59,7 \%)$ y 355 varones $(40,3 \%)$ de edades comprendidas entre 20 y 60 años los cuales fueron seleccionados al azar en instituciones universitarias y de salud (consultorio de carnet sanitario, escuela de educación física, Salud Universitaria).

De todas las medidas antropométricas relevadas se analizan en este trabajo las correspondientes a peso, talla, perímetros: braquial
(Pr.Braq.), cintura (Pr.Cint.), pierna (Pr.Pier.) y muslo (Pr.Mus.) y pliegues subcutáneos: tricipital (Pl.Tri.) y subescapular (Pl.Sub.).

Todas las mediciones antropométricas fueron realizadas por cuatro de los autores que poseen entrenamiento en esta práctica (dos de cada provincia, Jujuy: RC e IFB y Catamarca: ND y DL), utilizando técnicas estándar (Lohman et al., 1988). La talla, los perímetros y los pliegues se registraron con precisión de $1 \mathrm{~mm}$ y el peso de $0,1 \mathrm{~kg}$. La medición del peso se realizó con balanza Tanita (Modelo TBF-300A) y la de la talla con antropómetro vertical. Los pliegues cutáneos se midieron utilizando adipómetro de Harpenden y los perímetros con cinta antropométrica flexible e inextensible. La edad fue determinada por la diferencia entre la fecha de nacimiento y la de observación, considerándose años cumplidos.

Los datos se agruparon por sexo y dos grupos de edad: 20-39 años y 40-60 años para facilitar la comparación con la referencia. Se calcularon medidas de posición (media) y dispersión (desvío estándar). El estado nutricional se calculó por sexos y grupos de edad de acuerdo al criterio de la World Health Organization (WHO, 2000).

Independientemente de la edad se calcularon los parámetros $\mathrm{L}, \mathrm{M}$ y S por el método de la máxima probabilidad penalizada usando el método LMS (Cole y Green, 1992). Para cada variable fueron calculados los centilos 5, 10, $25,50,75,90$ y 95 aplicando la siguiente fórmula:

$$
\mathrm{C}=\mathrm{M}[1+\mathrm{LSZ}]^{1 / \mathrm{L}}
$$

donde L, M y $\mathrm{S}$ representan la asimetría (lambda), mediana (mu) y el coeficiente de variación (sigma) respectivamente de los valores estimados para cada sexo y edad y $\mathrm{Z}$ es el puntaje $\mathrm{z}$ correspondiente al centilo calculado (Cole, 1989; Cole y Green, 1992). La prueba Q fue utilizada para probar la bondad del ajuste. Los datos fueron procesados con el software LMS ChartMaker Pro.

A partir de la media de cada variable se realizaron comparaciones entre sexos y con la referencia CDC (2003-2006) (McDowell et al., 2008), correspondiente a adultos de 
EEUU de origen mexicano de la misma edad y sexo a través de la prueba $t$ de Student. Con dicha referencia se calcularon puntaje z promedio de cada variable por edad y sexo.

\section{RESULTADOS}

En la Tabla 1 puede observarse que la prevalencia más alta de obesidad $(48,1 \%)$ corresponde a varones de 40-60 años y que ésta aumenta con la edad en ambos sexos.

En las Tablas 2 a 5 se presentan la composición numérica de la muestra por sexo y grupos de edad, así como la media, el desvío estándar (DS), el puntaje $\mathrm{Z}$ promedio y los percentilos de las variables consideradas. Para todas las variables, excepto Pr.Mus. en ambos grupos de edad y Pl.Sub. en mayores de 40 años, se observan diferencias sexuales estadísticamente significativas. Los valores correspondientes a peso, talla y perímetros son superiores en los varones y los pertenecientes a los pliegues son mayores en las mujeres. Respecto de la referencia, en todas las variables y para los dos grupos de edad, las mujeres presentan valores significativamente inferiores, excepto para Pr.Mus. que aunque menor, no registra diferencias significativas $(\mathrm{p}<0,01$ para la talla de 20-39 años y Pl.Sub. entre 40-60 años; para el resto de las dimensiones $\mathrm{p}<0,001$ ). Las mujeres del NOA son menos pesadas, menos altas y con menor tejido adiposo que las de referencia, tanto en el abdomen como en las extremidades (Tablas 2, 3).

En varones de 20-39 años se observa una situación semejante a la anterior. Los hombres del NOA de este grupo etáreo presen-

TABLA 1. Número de individuos (N) por sexo y grupo de edad y porcentaje por estado nutricional según criterio OMS (2000)

\begin{tabular}{|c|c|c|c|c|c|c|c|c|c|c|}
\hline \multicolumn{2}{|c|}{$\begin{array}{l}\text { Estado Nutricional } \\
\qquad \mathrm{IMC}\left(\mathrm{kg} / \mathrm{m}^{2}\right)\end{array}$} & \multicolumn{2}{|c|}{$\begin{array}{l}\text { Bajo Peso } \\
\quad<18,5\end{array}$} & \multicolumn{2}{|c|}{$\begin{array}{l}\text { Rango Normal } \\
\geq 18,5 \mathrm{y} \leq 24,9\end{array}$} & \multicolumn{2}{|c|}{$\begin{array}{l}\text { Pre-Obesidad } \\
\geq 25,0 \text { y } \leq 29,9\end{array}$} & \multicolumn{2}{|c|}{$\begin{array}{l}\text { Obesidad } \\
\geq 30,0\end{array}$} & \multirow{2}{*}{$\mathrm{N}$} \\
\hline & & $\mathrm{N}$ & $\%$ & $\mathrm{~N}$ & $\%$ & $\mathrm{~N}$ & $\%$ & $\mathrm{~N}$ & $\%$ & \\
\hline \multirow{2}{*}{ Varones } & 20 a 39 años & 5 & 1,8 & 171 & 61,5 & 76 & 27,3 & 26 & 9,4 & 278 \\
\hline & 40 a 60 años & 0 & 0,0 & 16 & 20,7 & 24 & 31,2 & 37 & 48,1 & 77 \\
\hline \multirow{2}{*}{ Mujeres } & 20 a 39 años & 8 & 1,9 & 236 & 58,3 & 100 & 24,7 & 61 & 15,1 & 405 \\
\hline & 40 a 60 años & 1 & 0,8 & 37 & 30,6 & 47 & 38,8 & 36 & 29,8 & 121 \\
\hline
\end{tabular}

TABLA 2. Mujeres de 20-39 años. Número de individuos (N), Media,

Desvio estándar (DS), Media Pz y Percentilos $(P)$ de todas las variables

\begin{tabular}{cccccccccccc}
\hline Variable & N & Media & DS & Media Pz & P5 & P10 & P25 & P50 & P75 & P90 & P95 \\
\hline Peso (kg) & 405 & $62,2^{* * *}$ & 13,4 & $-0,5$ & 46,9 & 49,5 & 53,0 & 59,2 & 69,1 & 79,9 & 87,9 \\
Talla (cm) & 405 & $157,7^{* *}$ & 6,2 & $-0,1$ & 148,0 & 150,0 & 153,0 & 157,0 & 162,0 & 166,0 & 168,2 \\
Pr.Braq. (cm) & 366 & $27,2^{* * *}$ & 4,0 & $-0,8$ & 22,0 & 22,6 & 24,5 & 26,5 & 29,0 & 32,7 & 34,0 \\
Pr.Mus. (cm) & 264 & $51,9 \mathrm{~ns}$ & 6,4 & $-0,1$ & 43,2 & 44,9 & 47,6 & 51,0 & 56,0 & 60,7 & 64,0 \\
Pr.Pier. (cm) & 366 & $34,6^{* * *}$ & 3,8 & $-0,5$ & 29,8 & 30,5 & 32,0 & 34,0 & 36,0 & 39,9 & 42,0 \\
Pr.Cint. (cm) & 298 & $82,9^{* * *}$ & 12,1 & $-0,7$ & 66,0 & 68,9 & 74,4 & 80,7 & 90,0 & 99,1 & 104,0 \\
Pl.Tri. (mm) & 364 & $16,5^{* * *}$ & 5,2 & $-0,8$ & 8,3 & 11,0 & 13,0 & 16,0 & 20,0 & 22,4 & 25,0 \\
Pl.Sub. (mm) & 365 & $18,9 * * *$ & 6,9 & $-0,3$ & 9,2 & 11,0 & 14,0 & 18,0 & 23,0 & 28,0 & 32,0 \\
\hline
\end{tabular}

Nivel de significación prueba "t" NOA vs CDC: ns= no significativo; $* * \mathrm{p}<0,01 ; * * * \mathrm{p}<0,001$ 
TABLA 3. Mujeres de 40-60 años. Número de individuos (N), Media, Desvio estándar (DS), Media Pz y Percentilos $(P)$ de todas las variables

\begin{tabular}{cccccccccccc}
\hline Variable & $\mathrm{N}$ & Media & DS & Media Pz & P5 & P10 & P25 & P50 & P75 & P90 & P95 \\
\hline Peso (kg) & 121 & $66,5 * * *$ & 12,5 & $-0,5$ & 50,0 & 54,0 & 56,8 & 64,9 & 75,0 & 81,9 & 90,8 \\
Talla (cm) & 121 & $153,5 * * *$ & 6,3 & $-0,6$ & 144,1 & 146,6 & 148,8 & 153,0 & 157,3 & 163,0 & 165,0 \\
Pr.Braq. (cm) & 113 & $29,2 * * *$ & 3,6 & $-0,9$ & 24,3 & 25,0 & 27,0 & 29,0 & 31,0 & 34,0 & 35,3 \\
Pr.Mus. (cm) & 113 & 51,7 ns & 6,5 & $-0,1$ & 42,7 & 45,0 & 47,5 & 51,0 & 55,0 & 60,0 & 64,3 \\
Pr.Pier. (cm) & 113 & $35,1^{* * *}$ & 3,7 & $-0,6$ & 31,0 & 31,2 & 32,5 & 34,5 & 37,0 & 41,0 & 42,0 \\
Pr.Cint. (cm) & 121 & $89,3 * * *$ & 10,9 & $-0,5$ & 73,6 & 75,4 & 81,0 & 88,5 & 95,0 & 106,0 & 109,0 \\
P1.Tri. (mm) & 111 & $17,8^{* * *}$ & 4,9 & $-0,9$ & 10,6 & 12,0 & 15,0 & 17,0 & 20,0 & 25,0 & 27,0 \\
Pl.Sub. (mm) & 113 & $21,7 * *$ & 6,5 & $-0,4$ & 12,0 & 13,4 & 17,0 & 22,0 & 26,0 & 31,6 & 32,3 \\
\hline
\end{tabular}

Nivel de significación prueba “t” NOA vs CDC: $n s=$ no significativo; $* * p<0,01 ; * * * p<0,001$

taron valores significativamente menores en talla, peso, perímetros y pliegues respecto a los de referencia. En el grupo de edad de 4060 años se observan mayores valores en $P$, Pr.Mus., Pl.Tri. y Pl.Sub. aunque sin diferencias significativas. Los varones de este grupo son más pesados y presentan mayor cantidad de tejido adiposo tricipital y subescapular. Las otras variables se comportan del mismo modo que en el grupo de edad de 20-39 años (Tablas 4, 5).
Todas las variables analizadas tienen valores de puntaje $\mathrm{z}$ negativos entre $0 \mathrm{y}-1$ desvío estándar respecto a la referencia. Solamente están exceptuadas algunas variables, como P, Pr.Mus., Pl.Tri. y Pl.Sub. en la serie de varones mayores de 40 años. En estos casos el puntaje $\mathrm{z}$ tiene valores positivos entre 0 y 0,5 respecto a la referencia.

En las Figuras 2 a 9 se presentan los percentilos suavizados de cada variable por sexo, obtenidos con el método LMS. En el caso del

TABLA 4. Varones de 20-39 años. Número de individuos (N), Media, Desvío estándar (DS), Media Pz y Percentilos $(P)$ de todas las variables

\begin{tabular}{cccccccccccc}
\hline Variable & $\mathrm{N}$ & Media & DS & Media Pz & P5 & P10 & P25 & P50 & P75 & P90 & P95 \\
\hline Peso (kg) & 278 & $70,9 * * *$ & 11,9 & $-0,4$ & 55,3 & 58,6 & 63,2 & 68,7 & 76,4 & 84,8 & 93,2 \\
Talla (cm) & 278 & $168,9 * *$ & 8,1 & $-0,2$ & 154,0 & 159,0 & 164,4 & 169,0 & 174,0 & 179,0 & 183,0 \\
Pr.Braq. (cm) & 272 & $28,6^{* * *}$ & 2,9 & $-0,8$ & 24,0 & 25,1 & 27,0 & 28,5 & 30,2 & 32,1 & 34,0 \\
Pr.Mus. (cm) & 145 & $51,4 * * *$ & 5,0 & $-0,3$ & 43,0 & 44,8 & 47,5 & 51,0 & 55,0 & 58,0 & 59,5 \\
Pr.Pier. (cm) & 273 & $35,2^{* * *}$ & 3,0 & $-0,6$ & 30,1 & 32,0 & 33,5 & 35,0 & 36,5 & 39,0 & 40,2 \\
Pr.Cint. (cm) & 150 & $88,3^{* * *}$ & 10,1 & $-0,4$ & 74,0 & 76,0 & 81,9 & 87,0 & 94,0 & 102,0 & 106,5 \\
Pl.Tri. (mm) & 271 & $12,8^{* *}$ & 4,7 & $-0,2$ & 6,0 & 8,0 & 10,0 & 12,0 & 15,0 & 19,0 & 21,2 \\
Pl.Sub. (mm) & 272 & $16,4 * * *$ & 5,7 & $-0,2$ & 9,0 & 10,0 & 12,0 & 15,0 & 20,0 & 25,0 & 27,0 \\
\hline
\end{tabular}

Nivel de significación prueba "t" NOA vs CDC: **p<0,01; ***p $<0,001$ 
TABLA 5. Varones de 40-60 años. Número de individuos (N), Media, Desvío estándar (DS), Media Pz y Percentilos $(P)$ de todas las variables

\begin{tabular}{cccccccccccc}
\hline Variable & N & Media & DS & Media Pz & P5 & P10 & P25 & P50 & P75 & P90 & P95 \\
\hline Peso (kg) & 77 & $82,9 \mathrm{~ns}$ & 17,3 & 0,1 & 56,0 & 59,5 & 70,0 & 81,5 & 97,6 & 107,2 & 111,2 \\
Talla (cm) & 77 & $165,3 * * *$ & 7,7 & $-0,8$ & 150,9 & 153,8 & 159,9 & 166,0 & 169,5 & 177,0 & 178,2 \\
Pr.Braq. (cm) & 76 & $31,0^{* * *}$ & 3,4 & $-0,7$ & 24,8 & 26,5 & 29,0 & 30,3 & 33,1 & 35,5 & 37,0 \\
Pr.Mus. (cm) & 76 & $52,0 \mathrm{~ns}$ & 4,9 & 0,1 & 44,4 & 46,0 & 48,0 & 51,6 & 55,0 & 57,0 & 60,3 \\
Pr.Pier. (cm) & 76 & $36,3 * * *$ & 3,8 & $-0,5$ & 30,8 & 32,0 & 33,2 & 35,8 & 39,0 & 42,1 & 43,1 \\
Pr.Cint. (cm) & 77 & $98,6 \mathrm{~ns}$ & 11,4 & $-0,1$ & 81,0 & 81,1 & 93,0 & 99,7 & 107,0 & 112,9 & 115,4 \\
Pl.Tri. (mm) & 76 & $13,9 \mathrm{~ns}$ & 5,3 & 0,1 & 5,8 & 7,4 & 10,0 & 13,0 & 17,0 & 20,0 & 22,1 \\
Pl.Sub. (mm) & 76 & $20,9 \mathrm{~ns}$ & 5,7 & 0,1 & 11,2 & 14,0 & 17,0 & 21,0 & 24,0 & 29,3 & 30,3 \\
\hline
\end{tabular}

Nivel de significación prueba " $\mathrm{t}$ " NOA vs CDC: $\mathrm{ns}=$ no significativo; $* * \mathrm{p}<0,01 ; * * * \mathrm{p}<0,001$

peso de los varones tiende a aumentar con la edad hasta los 50 años, para luego descender suavemente. En cambio en las mujeres aumenta persistentemente hasta los 40 años y luego se mantiene estable hasta los 60 años (Fig. 2). La talla en cambio, tiende a disminuir con la edad en ambos sexos (Fig. 3). El Pr.Braq. manifiesta un comportamiento semejante en ambos sexos, con un aumento progresivo hasta los 45 años en los varones y aproximadamente los 42 años en las mujeres, para luego descender más bruscamente en los varones (Fig. 4). La trayectoria del Pr.Mus. es diferente según el sexo. En los varones desciende desde los 20 años hasta los 30 años, luego vuelve a aumentar hasta alcanzar su máximo alrededor de los 44 años y luego desciende ligeramente. En las mujeres también desciende desde los 20 a 30 años, luego se incrementa levemente hasta los 39 años y a partir de esa edad desciende paulatinamente hasta los 60 años (Fig. 5).

La trayectoria del Pr.Pier. en varones muestra una leve disminución hasta los 28 años, edad a partir de la cual se incrementa paulatinamente
A

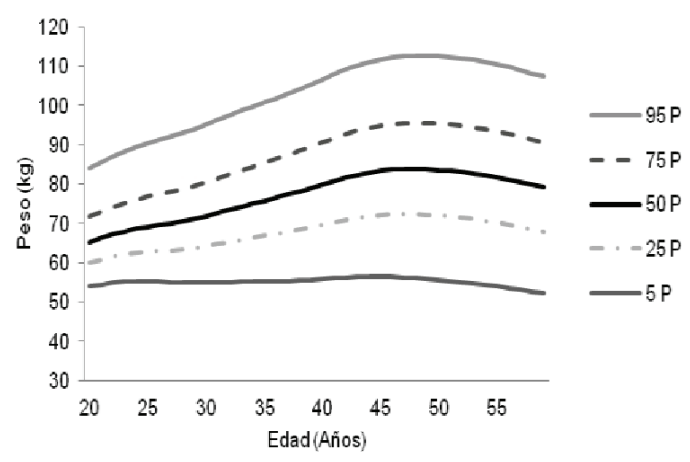

B

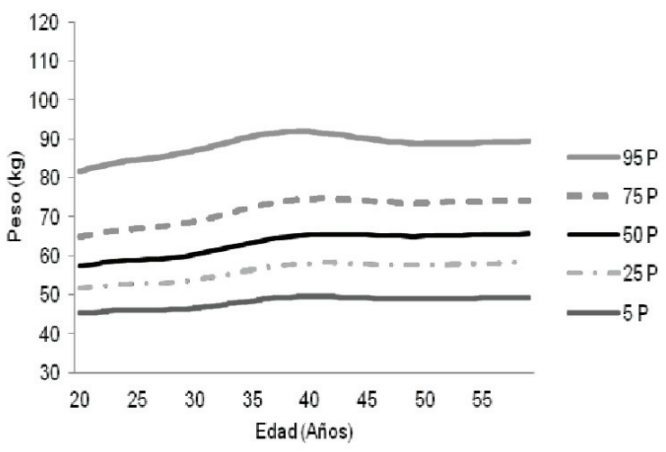

Fig. 2. Percentilos de Peso entre 20 y 60 años: A, varones; B, mujeres. 
A

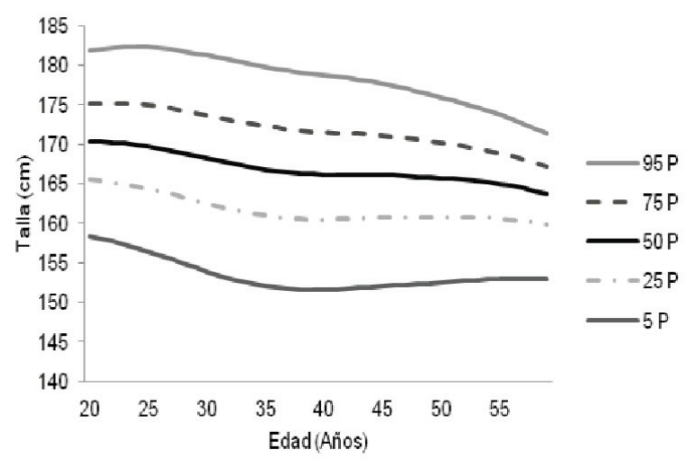

B

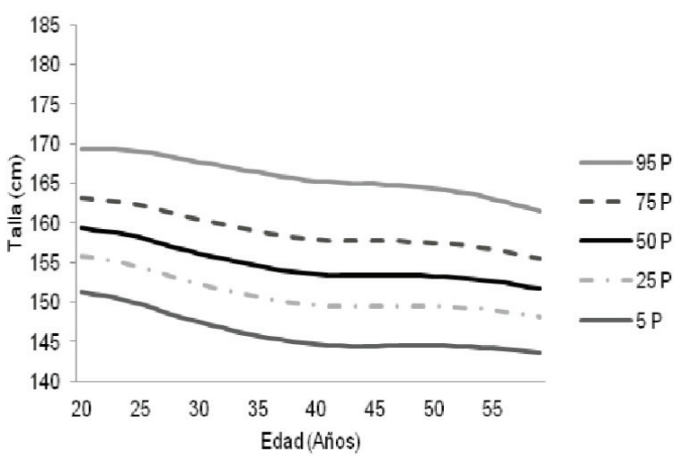

Fig. 3. Percentilos de Talla entre 20 y 60 años: A, varones; B, mujeres.

A

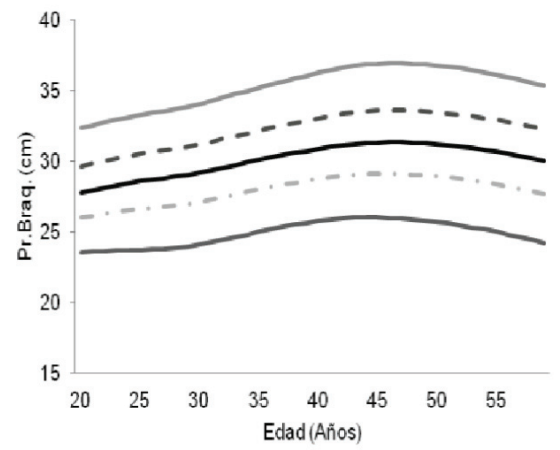

B

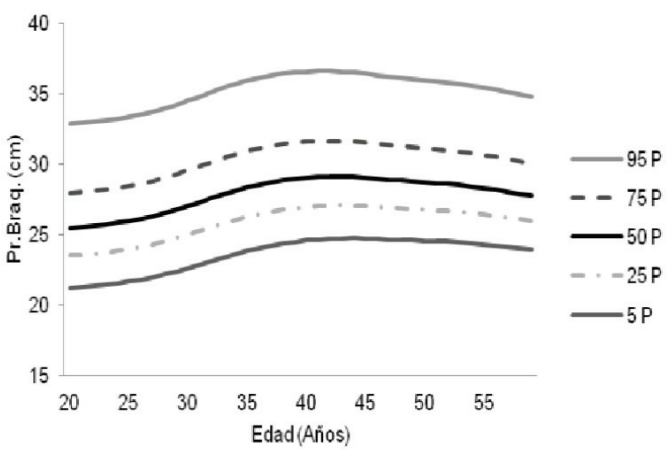

Fig. 4. Percentilos de Perímetro Braquial (Pr.Braq.) entre 20 y 60 años: A, varones; B, mujeres.

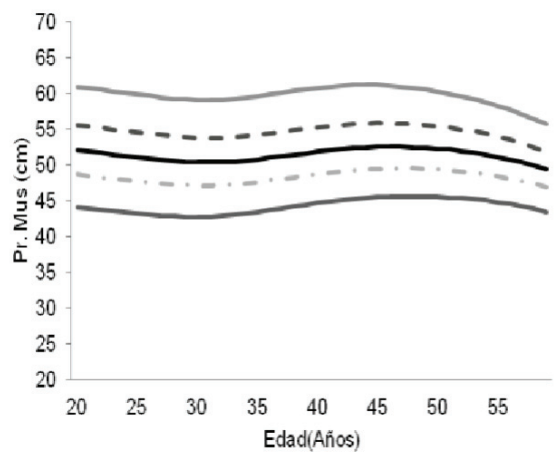

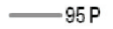
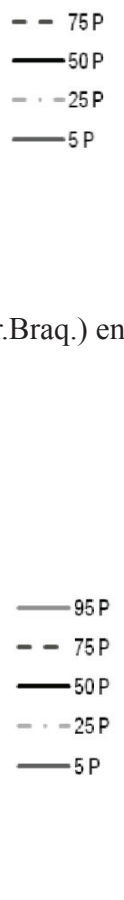

B

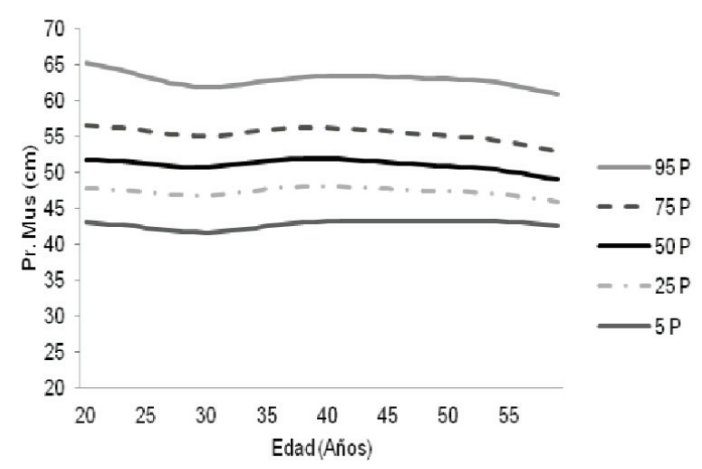

Fig. 5. Percentilos de Perímetro del Muslo (Pr.Mus.) entre 20 y 60 años: A, varones; B, mujeres.

hasta los 44 años decayendo luego ligeramente hasta los 60 años. En mujeres en cambio, aumenta de manera paulatina hasta los 40 años, se mantiene estable hasta los 50 años para declinar luego levemente hasta los 60 años (Fig. 6). En varones, el Pr.Cint. aumenta con la edad, pero a partir de la quinta década tiende a disminuir paulatinamente. En las mujeres se incrementa hasta los 40 años, se mantiene estable hasta los 49 años y luego aumenta ligeramente (Fig. 7). El Pl.Tri. en ambos sexos, se caracteriza por aumentos y disminuciones leves y sucesivas (Fig. 
8). El Pl.Sub. en varones aumenta desde los 20 años, alcanza su valor máximo a los 50 años y luego comienza a declinar. En las mujeres este pliegue aumenta hasta los 40 años y luego dis- minuye levemente hasta los 60 años (Fig.9).

DISCUSIÓN

Los resultados obtenidos fueron comparados
A

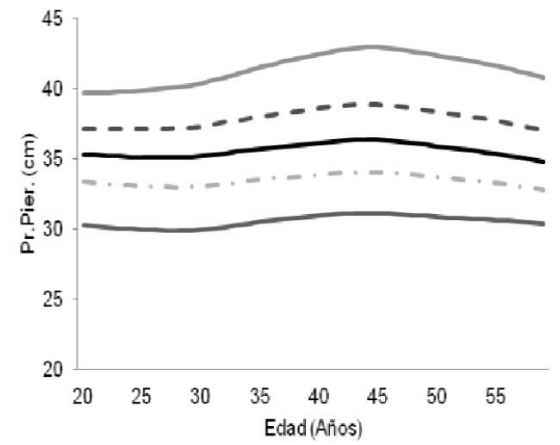

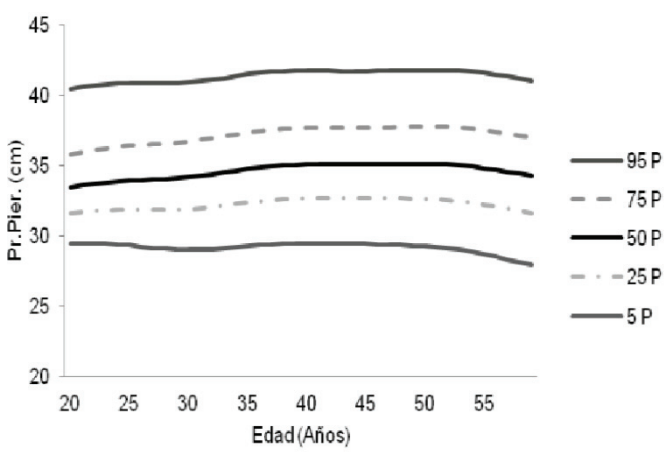

Fig. 6. Percentilos de Perímetro de la Pierna (Pr.Pier.) entre 20 y 60 años: A, varones; B, mujeres.

A

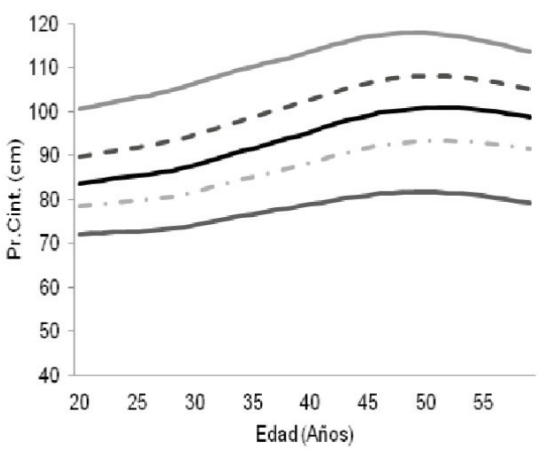

B

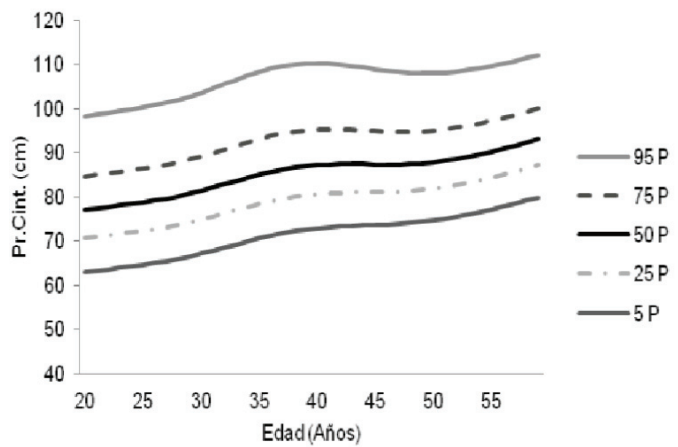

Fig. 7. Percentilos de Perímetro de la Cintura (Pr.Cint.) entre 20 y 60 años: A, varones; B, mujeres.

A

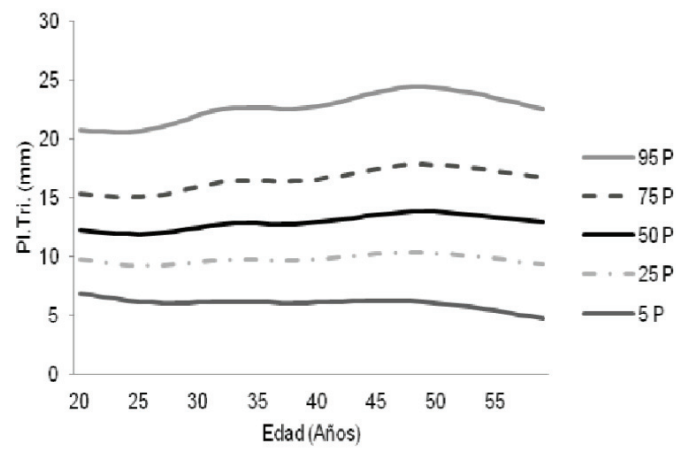

B

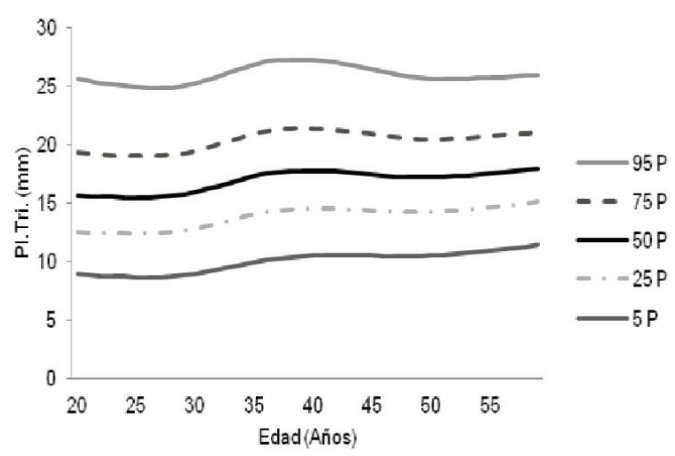

Fig. 8. Percentilos de Pliegue Subcutáneo Tricipital (Pl.Tri.) entre 20 y 60 años: A, varones; B, mujeres. 


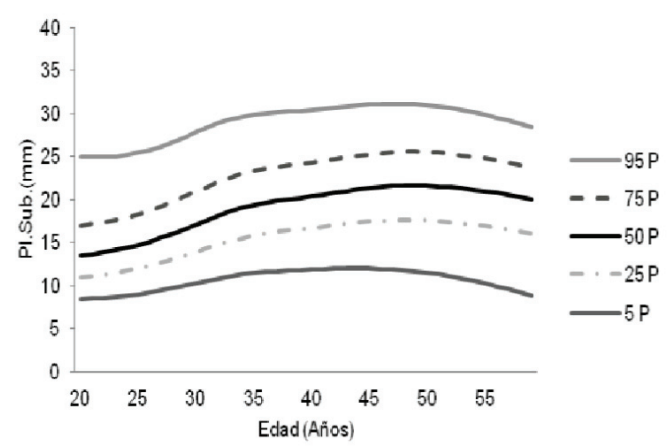

B

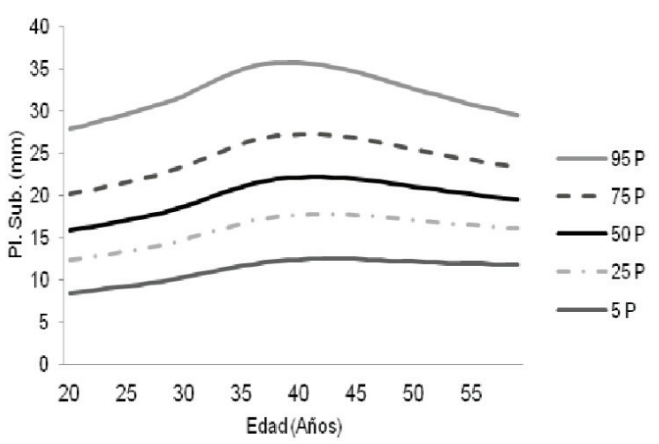

Fig. 9. Percentilos de Pliegue Subcutáneo Subescapular (Pl.Sub.) entre 20 y 60 años: A, varones; B, mujeres.

con una referencia internacional biológica y culturalmente relacionada, puesto que no se dispone de un antecedente antropométrico semejante para poblaciones del NOA ni de Argentina. La referencia utilizada corresponde a una muestra de población civil y no institucionalizada de Estados Unidos (norteamericanos de origen mexicano), que fue relevada en dos períodos: 20032004 y 2005-2006 (National Center for Health Statistics 2005, 2007). Es decir que se trata de una muestra que refleja la epidemia de obesidad (IMC $\geq 30)$ en Estados Unidos, donde la prevalencia en 2007-2008 para individuos de origen hispánico, independientemente del sexo, fue del $37,9 \%$ en sujetos $\geq 20$ años y las correspondientes a sobrepeso (IMC $\geq 25$ ) del $77,5 \%$ (Flegal et al., 2010). En la provincia de Jujuy fueron informadas prevalencias de sobrepeso que, de acuerdo a la altura sobre el nivel del mar oscilaron en varones entre $40 \%$ - $43 \%$ y en mujeres entre $29 \%$ $35 \%$, mientras que las prevalencias de obesidad oscilaron entre $22 \%-27 \%$ y entre $20 \%-32 \%$, respectivamente (Bejarano et al., 2013).

Siempre en relación al exceso de peso para el total del país, la Sociedad Argentina de Nutrición informó que en el año 2003 el 29\% de la población presentaba sobrepeso y el $27 \%$ obesidad (De Girolami, 2003) y según la Encuesta Nacional de Nutrición y Salud (ENNyS) (Ministerio de Salud de la Nación, 2007) el 22,7\% de las mujeres entre 10 y 49 años tenía sobrepeso, mientras que el $15,3 \%$ presentaba obesidad, correspondiendo al NOA una prevalencia de $\mathrm{S}+\mathrm{O}$ (sobrepeso más obesidad) del $39,7 \%$, superando al total del país (38\%) y sobrepasada sólo por la hallada en la región Patagonia (42,2\%).
Las prevalencias más altas de sobrepeso $(38,8 \%)$ y obesidad $(48,1 \%)$ halladas en el presente estudio, se presentaron en mujeres y varones de 40-60 años, mostrando en ambos sexos aumento con la edad. Otros autores han descrito esta misma tendencia. Así, el trabajo realizado por De Sereday et al. (2004) en la región central de Argentina y los resultados reportados por Elgart et al. (2010) en un análisis de datos retrospectivos provenientes de estadísticas nacionales, han informando un aumento progresivo hasta los 64 años de edad para la obesidad y hasta los 49 años para el sobrepeso. Aunque en general las mujeres suelen tener mayor sobrepeso/obesidad (Braguinsky, 2002), algunas investigaciones realizadas, especialmente en España (Aranceta et al., 2003; Montero et al., 2004) y en nuestro país (Carbajal et al., 2001) indicarían que esta tendencia se está invirtiendo, convirtiendo a los varones adultos en un nuevo grupo de riesgo. Los resultados obtenidos en este estudio confirmarían parcialmente tal tendencia, ya que considerando los estados de malnutrición conjuntamente y ambos grupos etáreos, la prevalencia es mayor en los hombres de edad más avanzada $(79,3 \%)$ que en las mujeres $(68,6 \%)$, mientras que en el grupo de 20 a 39 años las mujeres son las que alcanzan una prevalencia mayor $(39,8 \%)$ que la registrada en varones $(36,7 \%)$. Este hecho se corresponde en los varones de edad más avanzada con valores de peso, perímetro del muslo y pliegues mayores, aunque sin alcanzar diferencias significativas respecto a la referencia utilizada. Las poblaciones del NOA en relación a las de la referencia, tienen en ambos grupos etáreos y en ambos sexos me- 
nor talla, con diferencias mayores en el grupo de edad más avanzada (más de $4 \mathrm{~cm}$ ) asimismo, presentan menor tejido adiposo, a excepción del grupo etáreo de 40-60 años. Las pequeñas diferencias a favor de los varones mayores de 40 años pueden deberse a un efecto de muestreo teniendo en cuenta que en esta serie el número efectivo es sensiblemente inferior al de los otros grupos analizados.

Argentina presenta un estado avanzado de transición demográfica, epidemiológica y nutricional con características particulares y una gran heterogeneidad dentro de las provincias y entre las regiones (Lomaglio, 2012). Las poblaciones analizadas de alto componente indígena, están en plena transición nutricional y tal como han señalado otros investigadores (Popkin, 2008) pueden presentar mayor riesgo de obesidad una vez alcanzada la última fase de modernización de la dieta. Concretamente para Jujuy y Catamarca, Mesa et al. (2012) concluyeron que el NOA se encuentra en una fase avanzada de transición nutricional, en donde se mezclan alimentos tradicionales con otros mucho más elaborados. Estas poblaciones se caracterizan por tener una dieta monótona, con elevado consumo de carne vacuna, hidratos de carbono, grasas saturadas, azúcares y alimentos procesados y bajo consumo de legumbres y frutas (Mesa et al., 2012; Bejarano et al., 2013). De acuerdo a Mesa et al. (2012) sobre un total de 515 personas adultas de las provincias de Jujuy y Catamarca, más del $25 \%$ de los varones y mujeres presentaron dieta deficiente y aproximadamente un 15\% hábitos alimentarios no recomendables.

El análisis de las variables antropométricas, entre los 20 y 60 años de edad, en adultos del NOA da cuenta de la existencia de diferencias sexuales esperadas, pero también de modificaciones de la adiposidad a lo largo de la ontogenia con un compromiso fluctuante del tejido adiposo en la composición corporal de varones y mujeres. La magnitud de las modificaciones de la adiposidad en función del sexo y la edad como resultado de la interacción genético-ambiental tiene una gran importancia para comprender los factores condicionantes de la obesidad en las distintas etapas de la ontogenia. En tal sentido en el trabajo de Elgart et al. (2010) se informó una fuerte asociación entre frecuencia de sobrepeso/obesidad y niveles de instrucción y socioeconómico. Así, por ejemplo, el 16,2\% de los obesos y el 15,4\% de las personas con sobrepeso tenían algún tipo de necesidades básicas insatisfechas. Los antecedentes sobre el estado sociosanitario de las poblaciones del NOA que permitan interpretar y contextualizar el perfil antropométrico aquí descripto son escasos. La Encuesta Nacional sobre Factores de Riesgo (ENFR) para enfermedades no transmisibles, llevada a cabo en el 2005 y el 2009 por el INDEC y el Ministerio de Salud, proporcionan una serie de indicadores propuestos por la Organización Panamericana de la Salud (OPS) que permiten prever la carga de enfermedad y mortalidad atribuida a este tipo de patología en adultos (Ferrante y Virgolini, 2007; Ferrante et al., 2011). Si se analiza la evolución de los indicadores de riesgo entre 2005 y 2009 en el NOA presentados en la Tabla 6 se observa que algunos de ellos, como los correspondientes a presión arterial y colesterol elevados aumentaron en el NOA sensiblemente más que en el resto del país, asimismo para la glucemia elevada, mientras se observó un valor similar en ambas encuestas en el NOA, en el resto del país disminuyó. Como puede observarse también, las poblaciones del NOA presentan en relación al resto del país, menor calidad de vida y menor actividad física, elevada presión arterial, glucemia y diabetes, mayor obesidad, colesterol y consumo de tabaco y realizan menos actividades para prevención de enfermedades crónicas no transmisibles tales como el control de presión arterial, colesterol y glucemia. Tales observaciones reflejan, no sólo la desigualdad del NOA respecto del resto del país, sino también del incremento en los niveles de riesgo de la población en estudio. Si bien la información que proporciona la ENFR tiene un carácter autorreferencial, es decir que no está basada en mediciones ni análisis, la misma coincide en general con otros antecedentes sobre las poblaciones del NOA que indican altas prevalencias de obesidad y hábitos alimentarios.

Los resultados obtenidos en este trabajo son válidos para esta muestra de individuos con edades comprendidas entre los 20 y 60 años y los mismos constituyen un aporte al conocimiento de las características antropométricas de poblaciones contemporáneas del NOA en el contexto de la epidemia de obesidad. El análisis realizado implicó la desagregación de la muestra en dos 
TABLA 6. Distribución porcentual (Intervalo de Confianza 95\%) de indicadores de riesgo cardiovascular a nivel nacional y del NOA en la Encuesta Nacional de Factores de Riesgo 2005 y 2009

\begin{tabular}{ccccc}
\hline Indicador & Nacional & NOA & Nacional & NOA \\
& 2005 & 2005 & 2009 & 2009 \\
\hline Calidad de vida regular o mala & $19,9(19,0-20,7)$ & 28,7 & $19,2(18,5-20,0)$ & - \\
Baja actividad física & $46,2(44,7-47,5)$ & 40,9 & $54,9(53,9-55,9)$ & 44,3 \\
Obesidad & $14,6(13,8-15,4)$ & 16,0 & $18,0(17,4-18,7)$ & 18,8 \\
Control de la presión arterial el último año & $68,4(67,2-69,4)$ & 63,7 & $81,4(80,6-82,1)$ & 77,5 \\
Presión arterial elevada (una o más veces) & $34,4(33,3-35,5)$ & 23,4 & $34,8(34,0-35,7)$ & 35,1 \\
Control de colesterol & $72,8(71,3-74,4)$ & 42,5 & $76,6(75,5-77,6)$ & 68,0 \\
Colesterol elevado & $27,8(26,5-29,0)$ & 33,1 & $28,1(28,2-30,1)$ & 35,7 \\
Control de glucemia & $69,3(68,0-70,5)$ & 60,1 & $75,7(74,8-76,6)$ & 66,6 \\
Diabetes o glucemia elevada & $11,9(10,9-12,8)$ & 10,6 & $9,6(9,1-10,1)$ & 10,5 \\
Consumo de tabaco (18 años y más) & $29,7(28,6-30,8)$ & 31,8 & $27,1(26,3-27,9)$ & 26,2 \\
Consumo regular de riesgo de alcohol & $9,6(8,9-10,2)$ & 6,6 & $10,7(10,1-11,3)$ & - \\
\hline
\end{tabular}

grupos etáreos, como consecuencia de la cual el grupo de varones de edad más avanzada quedó integrado por un número de individuos muy inferior al resto de los grupos. Por ello no puede descartarse que, las observaciones realizadas en este último grupo tengan un sesgo, producto del efecto del muestreo. No obstante estas limitaciones los resultados también podrían extrapolarse a otras poblaciones del NOA.

\section{CONCLUSIONES}

La muestra de adultos analizada presenta alta prevalencia de exceso de peso, con un mayor compromiso en individuos mayores a 40 años. Respecto a las referencias del CDC, varones y mujeres presentan en general menores valores en las dimensiones de peso corporal y talla y tienen menor componente graso a nivel central y periférico.

\section{AGRADECIMIENTOS}

A las personas voluntarias que participaron del estudio y a las autoridades provinciales y nacionales de las provincias de Jujuy y Catamarca que prestaron su apoyo para el relevamiento de datos.

\section{LITERATURA CITADA}

Agrelo F, Pascual LR, Lobo B, Sabulsky J. 1999. Talla final de mujeres de Córdoba, Argentina, y exploración de la tendencia secular (1978-1988). Rev Panam Salud Publica 5(1):17-22. doi:10.1590/S102049891999000100003

Aranceta J, Pérez Rodrigo C, Serra Majem L, Ribas Barba L, Quiles Izquierdo J, Vioque J, Tur Marí J, Mataix Verdú J, Llopis González J, Tojo R, Foz Sala M. 2003. Grupo colaborativo para el estudio de la obesidad en España. Prevalencia de la obesidad en España: resultados del estudio SEEDO 2000. Med Clin (Barc) 120:608-612. doi:10.1016/S0025-7753(03)73787-7

Bazán AR. 1986. Historia del Noroeste Argentino. Buenos Aires: Editorial Plus Ultra.

Bejarano IF, Alfaro EL, Torrejón I, Pacheco JL, Mesa MS, López Parra AM, Lomaglio DB, Marrodán MD, Dipierri JE. 2013. Composición corporal y adiposidad en adultos jujeños de distintos niveles altitudinales. Rev Arg Antrop Biol 15(1):29-36.

Bejarano I, Dipierri JE, Ocampo SE. 1996. Variación regional de la tendencia secular de la talla adulta masculina en la Provincia de Jujuy. Rev Arg Antrop Biol 1(1):718.

Bejarano IF, Dipierri JE, Andrade A, Alfaro EL. 2009. Geographic altitude, surnames, and height variation of Jujuy (Argentina) conscripts. Am J Phys Anthropol 138(2):158-163. doi:10.1002/ajpa.20915

Boleda M. 1998. Ciudades del Noroeste Argentino. Buenos Aires: Alianza Editorial.

Bolsi A, Longhi F, Paolasso P. 2009. Pobreza y mortalidad infantil en el norte grande argentino. Un aporte para la formulación de políticas públicas. Cuadernos Geográfi$\cos 45: 231-261$. 
Braguinsky J. 2002. Prevalencia de obesidad en América Latina. Anales Sis San Navarra 25(1):109-115.

Butte NF, Garza C, de Onis M. 2007. Evaluation of the feasibility of international growth standards for schoolaged children and adolescents. J Nutr 137(1):153-157.

Carnese FR, Pucciarelli HM. 2007. Investigaciones antropobiológicas en Argentina, desde la década de 1930 hasta la actualidad. Relaciones XXXII:243-280.

Carbajal HA, Salazar MR, Riondet B, Rodrigo HF, Quaini SM, Rechifort V, Aizpurua M, Echeverría RF. 2001. Associated variables to hypertension in a region of Argentina. Medicina 61(6):801-809.

Cole TJ. 1989. The British, American NCHS, and Dutch weight standards compared using the LMS method. Am J Hum Biol 1(4):397-408. doi:10.1002/ ajhb. 1310010402

Cole TJ, Green PJ. 1992. Smoothing reference centile curves: the LMS method and penalized likelihood. Statist Med 11(10):1305-1319. doi:10.1002/sim.4780111005

De Girolami D. 2003. Descripción y análisis del índice de masa corporal y categoría pondoestatural por edades en un registro de 10.338 individuos de la República Argentina. Grupo de trabajo de valoración nutricional de la SAN. Actual Nutr 4(2):12-17.

De Onis M, Habicht JP. 1996. Anthropometric reference data for international use: recommendations from a World Health Organization Expert Committee. Am J Clin Nutr 64(4):650-658.

De Sereday MS, González C, Giorgini D, De Loredo L, Braguinsky J, Cobeñas C, Libman C, Tesone C. 2004. Prevalence of diabetes, obesity, hipertension and hiperlipidemia in the central area of Argentina. Diabetes Metab 30:335-339. doi:10.1016/S1262-3636(07)70125-8

Del Valle M, Vuao E, Gregori G. 2007. Síndrome metabólico en adultos sanos que concurren a un servicio de medicina preventiva. Archivos de Medicina Familiar y General 4(2):3-11.

Dipierri JE, Alfaro EL, Bailliet G, Bravi C, Albeck ME, Muzzio M, Ramallo V, Motti JMB. 2011. Estructura genético-isonímica del noroeste argentino. BAG J Basic Appl Genet 22(2):4-16.

Elgart J, Pfirter G, Gonzalez L, Caporale J, Cormillot A, Chiappe ML, Gagliardino J. 2010. Obesidad en Argentina: epidemiologia, morbimortalidad e impacto económico. Rev Argent Salud Pública 1(5):6-12.

Feldstein CA, Akopian M, Olivieri AO, Kramer AP, Nasi M, Garrido D. 2005. A comparison of body mass index and waist-to-hip ratio as indicators of hypertension risk in an urban Argentine population: a hospital-based study. Nutr Metab Cardiovasc Dis 15(4):310-315. doi:10.1016/j.numecd.2005.03.001

Ferrante D, Virgolini M. 2007. Encuesta Nacional de Factores de Riesgo 2005: resultados principales. Prevalencia de factores de riesgo de enfermedades cardiovasculares en la Argentina. Rev Argent Cardiol 75:20-29.

Ferrante D, Linetzky B, Konfino J, King A, Virgolini M, Laspiur S. 2011. Encuesta Nacional de Factores de Riesgo 2009: evolución de la epidemia de enfermedades crónicas no transmisibles en Argentina. Estudio de corte transversal. Rev Argent Salud Pública 2(6):34-41.

Flegal KM, Carroll MD, Ogden CL, Curtin LR. 2010. Prevalence and trends in obesity among US adults, 1999-2008. JAMA 303(3):235-241. doi:10.1001/ jama.2009.2014

Hernández RE, Cardonnet LJ, Libman C, Gagliardino JJ. 1987. Prevalence of diabetes and obesity in an ur- ban population of Argentina. Diabetes Res Clin Pract 3(5):277-283. doi:10.1016/S0168-8227(87)80051-7

Lohman TG, Roche AF, Martorell R. 1988. Anthropometric standardization reference manual. Champaign: Human Kinetics.

Lomaglio DB. 2012. Transición nutricional y el impacto sobre el crecimiento y la composición corporal en el noroeste argentino (NOA). Nutr Clín Diet Hosp 32(3):30-35.

McDowell MA, Fryar CD, Ogden CL, Flegal KM. 2008. Anthropometric reference data for children and adults: United States, 2003-2006. Hyattsville: National Center for Health Statistics.

Mesa MS, Marrodán MD, Moreno-Romero S, Viera-Peixoto A, García González M, López Ejeda N, Bejarano IF, Pacheco JL, López Parra AM, Dipierri JE, Lomaglio DB. 2012. Nutrición y globalización: diversidad y calidad de la dieta en una población del Noroeste de Argentina (NOA). En: Turbón Borrega D, Fañanás Saura L, Rissech Badalló C, Rosa A, editores. Biodiversidad humana y evolución. Barcelona: Sociedad Española de Antropología Física y Universidad de Barcelona. p 109-111.

Ministerio de Salud de la Nación. Encuesta Nacional de Nutrición y Salud (ENNyS) 2005. Documento de resultados. 2007. Disponible en: http://datos.dinami.gov.ar/ produccion/nutricion/material/A1a.pdf

Montero P, Morales EM, Carvajal A. 2004. Valoración de la percepción de la imagen corporal mediante modelos anatómicos. Antropo 8:107-116.

National Center for Health Statistics. 2005. National Health and Nutrition Examination Survey, 2003-2004 examination files. Disponible en: http://www.cdc.gov/nchs/about/ major/nhanes/nhanes2003-2004/ exam03 04.htm. 2005.

National Center for Health Statistics. 2007. National Health and Nutrition Examination Survey, 2005-2006 examination files. Disponible en: http://www.cdc.gov/nchs/ about/major/nhanes/nhanes2005-2006/exam05 06 . htm. 2007.

Organización Mundial de la Salud (OMS). 1995. El estado físico: uso e interpretación de la antropometría. Informe de un Comité de Expertos de la OMS. Serie de Informes Técnicos $\mathrm{N}^{\circ} 854$.

Peláez E, Torres VE, Acosta RS, Massobrio E. 2010. Ingresos de los hogares y estado nutricional de adultos en una ciudad intermedia del interior de Argentina. Población y Salud en Mesoamérica 7(2). Disponible en: http://ccp. ucr.ac.cr/revista/. doi:10.15517/psm.v7i2.1086

Pontussi EP. 1995. Geografía del Noroeste argentino. Salta: Facultad de Ciencias Naturales. Universidad Nacional de Salta.

Popkin B. 2008. Will China's nutrition transition overwhelm its health care system and slow economic growth? Health Aff (Millwood) 27(4):1064-1076. doi:10.1377/ hlthaff.27.4.1064

Wang S, Ray N, Rojas W, Parra MV, Bedoya G, Gallo C, Poletti G, Mazzotti G, Hill K, Hurtado AM, Camrena B, Nicolini H, Klitz W, Barrantes R, Molina JA, Freimer NB, Bortolini MC, Salzano FM, Petzl-Erler ML, Tsuneto LT, Dipierri JE, Alfaro EL, Bailliet G, Bianchi NO, Llop E, Rothhammer F, Excoffier L, Ruiz-Linares A. 2008. Geographic patterns of genome admixture in Latin American mestizos. Plos One 4(3): 19. doi:10.1371/journal.pgen.1000037

World Health Organization (WHO). 2000. Obesity: preventing and managing the global epidemic. Geneva: Report of a WHO Consultation. WHO Technical Reports Series 894 